\title{
A dynamical approach to identify vertices' centrality in complex networks
}

\author{
Long Guo $^{\mathrm{a}, \mathrm{b}, *}$, Wen-Yao Zhang ${ }^{\mathrm{b}}$, Zhong-Jie Luo ${ }^{\mathrm{a}}$, Fu-Juan Gao ${ }^{\mathrm{a}}$, Yi-Cheng Zhang ${ }^{\mathrm{c}, \mathrm{b}, *}$ \\ a School of Mathematics and Physics, China University of Geosciences (Wuhan), 430074, Wuhan, China \\ b Department of Physics, University of Fribourg, CH1700, Fribourg, Switzerland \\ ${ }^{\mathrm{c}}$ Institute of Green and Intelligent Technology, Chinese Academy of Sciences, 400714, Chongqing, China
}

In this paper, we proposed a dynamical approach to assess vertices' centrality according to the synchronization process of the Kuramoto model. In our approach, the vertices' dynamical centrality is calculated based on the Difference of vertices' Synchronization Abilities (DSA), which are different from traditional centrality measurements that are related to the topological properties. Through applying our approach to complex networks with a clear community structure, we have calculated all vertices' dynamical centrality and found that vertices at the end of weak links have higher dynamical centrality. Meanwhile, we analyzed the robustness and efficiency of our dynamical approach through testing the probabilities that some known vital vertices were recognized. Finally, we applied our dynamical approach

Keywords:

Dynamical centrality DSA

The Kuramoto model

Complex network to identify community due to its satisfactory performance in assessing overlapping vertices. Our present work provides a new perspective and tools to understand the crucial role of heterogeneity in revealing the interplay between the dynamics and structure of complex networks.

\section{Introduction}

Recent decades have witnessed a vigorous development of the network science, which is an interdisciplinary academic field to understand the behavior of natural, social and technical systems under the fundamental framework of complex networks [1]. Empirical analysis shows that many real complex networks, where vertices represent the elementary units of a given system and links describe the interactions between units [2,3], exhibit some nontrivial properties, such as the heterogeneous nature of vertices (e.g., the power-law distribution of vertex's connectivity) and the community structure (also called clustering or module). Those properties demonstrate that heterogeneity means difference, which indicates that to identify the vital vertices has its remarkable role in analyzing the structure and dynamics of complex networks [4]. For example, in complex network with a clear community structure, vital vertices with higher betweenness centrality, which are called the overlapping vertices, belong to more than one com-

\footnotetext{
* Corresponding authors. Long Guo at: School of Mathematics and Physics, China University of Geosciences (Wuhan), 430074, Wuhan, China; Yi-Cheng Zhang at: Institute of Green and Intelligent Technology, Chinese Academy of Sciences, 400714, Chongqing, China.

E-mail addresses: guolong@cug.edu.cn (L. Guo),yi-cheng.zhang@unifr.ch (Y.-C. Zhang).
}

munity commonly. To identify the overlapping vertices effectively benefits the community detection [5-8]. Meanwhile, the influential vertices can be quantified by various indexes. Take degree centrality for example, vertices with larger degree have an ability to influence more other vertices. To monitor those influential vertices is helpful for the prediction and control of spreading dynamics $[9,10]$.

The vital vertices, which can be identified using the concept of centrality, are largely affected and reflected by the topological structure and dynamical pattern of the network to which they belong. A tremendous number of methods for centrality have been proposed and well studied mainly based on the local or global topological structure $[4,11]$, such as the degree centrality, K-core decomposition, betweenness centrality and eigenvector centrality. And those methods neglect the critical role of dynamical processes in identifying vertices' importance. Although some dynamics-based centrality has been studied [4,9,12-14], to identify the vital vertices effectively and efficiently from the dynamical perspective also remains a big challenge. Since the structure and dynamics are tightly coupled in complex networks, dynamics is fundamental in assessing the impact of vertices in global performance [15-18]. For example, the Kuramoto model shares different synchronization behaviors on the homogeneous and heterogeneous complex networks [19].

As is well known, synchronization is a collective phenomenon occurring in systems of mutual interaction between units and 
is ubiquitous in nature, society and technology [20]. And the emergence of synchronization patterns in these systems has been shown to be closely related to the underlying topology of interactions [21-23]. For example, community (a set of oscillators which are placed at the vertices) with highly density of interactions synchronizes more easily than that with sparse connections, which leads to the application of the Kuramoto model in detecting community and overlapping in complex networks [22-24]. In this paper, we focus on the analysis of the difference of the local synchronization paces and propose a dynamical approach to identify the importance of vertices in a complex network. The fundamental idea of our dynamical approach is that vertices' centrality is calculated based on the DSA. We analyze the robustness and efficiency of our dynamical approach through applying it to identify community in the PPIN, the DSN and the benchmark network. We find that our approach has powerful advantages of timely, robustness and efficiency to identify vital vertices, for instance the overlapping community, in complex networks.

\section{Methodology and analysis}

One of the simple paradigm to understand the synchronization phenomenon is the Kuramoto model $[25,26]$, which has been applied to study the synchronization patterns and to identify community in complex networks. Consider a network of $N$ vertices joined in pairs by $E$ links, which represent the interaction between vertices, for example, the acquaintance or collaborations between individual in social networks. In graph description, the network can be represented by means of a $N \times N$ connectivity matrix $A$, where $A_{i j}=1$ when vertices $i$ and $j$ are linked, and $A_{i j}=0$, otherwise. Each vertex, says $i$, is encoded a phase oscillator with the natural frequency $\omega_{i}$ and phase $\theta_{i}$. The phase $\theta_{i}$ evolves in time according to the Kuramoto model:

$$
\frac{d \theta_{i}(t)}{d t}=\omega_{i}+\frac{\lambda}{k_{i}} \sum_{j \in \Gamma_{i}} \sin \left(\theta_{j}(t)-\theta_{i}(t)\right)
$$

where, $\Gamma_{i}$ is the subgraph of vertex $i$ 's nearest neighbors, $k_{i}$ is the connectivity of vertex $i$ and $\lambda$ is the positive coupling strength between vertices. Note that vertices are represented by their corresponding oscillators in the following part of our paper. Many previous literatures show that there exists a critical coupling $\lambda_{c}$, above which synchronization emerges spontaneously $[25,26]$. We here realize the Kuramoto model on complex networks using the Runge-Kutta methods. Without lack of generality, the coupling strength $\lambda=2\left(>\lambda_{c}\right)$ is fixed, and the natural frequencies $\omega_{i} \mathrm{~s}$ and the initial phase $\theta_{i}$ s are chosen from a uniform distribution $g(x)$ with mean $\langle x\rangle=0$ in the interval $(-0.5,0.5)$ and $(-\pi, \pi)$, respectively.

The macroscopic complex order parameter, which describes the synchronized behavior of the whole system, is defined as:

$R(t) e^{i \Phi(t)}=\frac{1}{N} \sum_{j=1}^{N} e^{i \theta_{j}(t)}$

where $R(t)(0 \leq R(t) \leq 1)$ quantifies the extent of synchronization in a system of $N$ oscillators, and $\Phi(t)$ is the average phase of the system at time $t$. The larger the $R(t)$ is, the more oscillators tend to synchronize to a common phase. In the special case of $R(t)=1$, all oscillators share the same phase and the system reaches the coherence state. However, $R(t)$ does not give any further information about the synchronization behavior in terms of local cluster, which plays a crucial importance to understand the structural and dynamical role of heterogeneity in complex networks. For this reason,

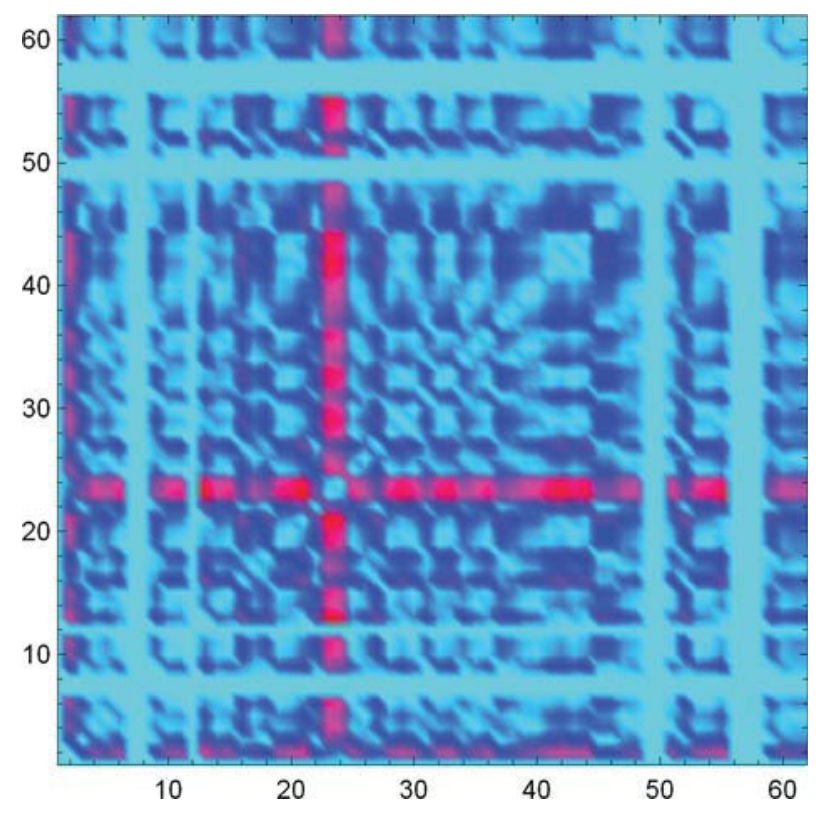

Fig. 1. (Color online.) The re-scaled DSA between pairs of oscillators in the DSN at the dynamical time $t=300$. The colors are a gradation between cyan $(0)$ and the red (1).

instead of considering the global order parameter, the real-valued local order parameter $r_{j}(t)$ at time $t$ is defined as follows:

$r_{j}(t) e^{i \Phi_{j}(t)}=\frac{1}{k_{j}+1} \sum_{l \in \Gamma_{j}^{\prime}} e^{i \theta_{l}(t)}$

where, $\Gamma_{j}^{\prime}$ is a subgraph including oscillator $j$ and its nearest neighbors, $\Phi_{j}(t)$ describes the average phase of oscillators in the given subgraph $\Gamma_{j}^{\prime}$, and $r_{j}(t)$ describes the extent of synchronization of the set of oscillators surrounding oscillator $j$ at time $t$. Further, the local order parameter $r_{j}(t)$, which represents the dynamical function of oscillator $j$, can be used to quantify the synchronization ability of oscillator $j$ at time $t$. Take a network with a clear community structure for example, oscillators belonging to the same community tend to share the same synchronization ability, and not necessarily equal for all communities [23]. While some oscillators often belong to more than one community and can be called the overlapping oscillators. Those overlapping oscillators will have a weak synchronization ability due to the limited from different communities. We here focus on the DSA among oscillators and do not consider the similar correlation between oscillators [22].

The DSA between oscillators, says $i$ and $j$, is written as,

$\Delta r_{i j}(t)=\left|r_{i}(t)-r_{j}(t)\right|$

And we operate a simple algebraic calculation to re-scale all those $\Delta r_{i j}(t)$ to fall in the range of $[0,1]$,

$\Delta^{\prime} r_{i j}(t)=\frac{r_{i j}(t)-r_{\min }(t)}{r_{\max }(t)-r_{\min }(t)}$

where, $r_{\min }(t)=\operatorname{Min}\left\{r_{i j}(t), \forall i, j\right\}$ and $r_{\max }(t)=\operatorname{Max}\left\{r_{i j}(t), \forall i, j\right\}$. Take a complex network with clear community structure for example, it is obvious that oscillators, says $i$ and $j$, in the same community have the similar synchronized ability with the smaller $\Delta^{\prime} r_{i j}(t)$ at time $t$.

In Fig. 1, we represent $\Delta^{\prime} r_{i j}(t)$ at $t=300$ for the DSN [27]. We can identify that the oscillators with No. 22 and 23 have a large gap about the re-scaled DSA with other oscillators, which clearly indicates that the two oscillators have a large probability to be 
the overlapping ones and play the bridge role among communities. Our preliminary result here demonstrates that the dynamical function of the re-scaled DSA plays a positive effect to reveal the underlying structural function directly.

Then, we define the quantity $\eta_{i}(t)$, which describes the average re-scaled DSA of oscillator $i$ in the system, as the below mathematical form,

$\eta_{i}(t)=\frac{1}{N} \sum_{j=1}^{N} \Delta^{\prime} r_{i j}(t)$

Analogy to the DSA, we also re-scaled $\eta_{i}(t)$ to fall in the range of $[0,1]$,

$\eta_{i}^{\prime}(t)=\frac{\eta_{i}(t)-\eta_{\min }(t)}{\eta_{\max }(t)-\eta_{\min }(t)}$

where, $\eta_{\min }(t)=\operatorname{Min}\left\{\eta_{i}(t), \forall i\right\}$ and $\eta_{\max }(t)=\operatorname{Max}\left\{\eta_{i}(t), \forall i\right\}$.

And then, we define the dynamical centrality of oscillator $i$ at time $t$ as follows

$\left\langle\eta_{i}^{\prime}\right\rangle_{t}=\frac{1}{t} \sum_{\tau=1}^{t} \eta_{i}^{\prime}(\tau)$

which is the average accumulative value of $\eta_{i}^{\prime}(t)$ and has a potential application to reveal oscillator $i$ 's topological status in a system at time $t$. In the special case, oscillator with the connectivity $k=1$ is a leaf and its $\left\langle\eta^{\prime}\right\rangle_{t}$ is set to zero in the given network. There are two folds of advantage of our present approach: one is that we build the simple correlation between dynamics and structure, which shows that difference (or heterogeneity) plays an important role in revealing the interplay between the dynamics and structure of complex system; the other is that the definition of $\left\langle\eta_{i}^{\prime}\right\rangle_{t}$ allows us to trace the time evolution of each oscillator's dynamical status and to identify vertices' topological status from the dynamical perspective timely. Our dynamical approach provides a practical way to unveil the potential interactions between vertices through their dynamical behaviors, such as the behavior of sending and receiving letters in the email networks and the process of the biochemical reaction in the protein-protein interaction network.

In Fig. 2, we represent $\left\langle\eta_{i}^{\prime}\right\rangle_{t}$ of each oscillator in the four different complex networks: the DSN with the size $N=62$, the PPIN with $N=163$, the network of coauthorships between $N=379$ scientists whose research centers on the top of networks [28], the benchmark network with $N=128$, the college football network with $N=115$ [29] and the Barabasi-Albert scale-free network (BASFN) with $N=100$, at time $t=300$. The benchmark network is built with $N=128$ vertices divided into four communities of 32 vertices each. Vertices in the same community are linked with probability $p_{i n}$ and vertices in different communities are linked with probability $p_{\text {out }}[29]$. And the BASFN is constructed based on two basic principles of growth and preferential attachment [30]. The value of each oscillator's $\left\langle\eta_{i}^{\prime}\right\rangle_{t}$ is proportional to the radius of the corresponding circle. The difference among oscillators' $\left\langle\eta_{i}^{\prime}\right\rangle_{t}$ in BASFN is smaller than that in the other five networks with a clear community structure. While the oscillators with higher value of $\left\langle\eta_{i}^{\prime}\right\rangle_{t}$ mostly lie on the marginal of community in complex network with a clear community structure, such as the DSN, the PPIN [31], the network of coauthorships between scientists [28] and the benchmark network with parameters $p_{\text {in }}=0.05$ and $p_{\text {out }}=0.005$. Those oscillators with higher value of $\left\langle\eta_{i}^{\prime}\right\rangle_{t}$ called the overlapping oscillators play the bridge role in connecting motifs.

Before analyzing the application of our dynamical approach, we should study the robustness of our approach due to the presence of initial dynamical noise and the stochastic effects. We realized the Kuramoto model 50 times independently in the DSN and the
PPIN, respectively. For each dynamical time $t$, we sort all the oscillators from big to small according to their $\left\langle\eta_{i}^{\prime}\right\rangle_{t}$. We then analyze the probabilities that each oscillator falls in the top ten $P_{10}$ and in the top twenty $P_{20}$ evolve as the dynamical time $t$ elapses. In Fig. 3, we find that $P_{10}$ and $P_{20}$ of those given oscillators, such as the oscillator 120 in the PPIN and the oscillator 21 in the DSN, reach their own steady values when $t>100$ respectively, which certifies the robustness of our dynamical approach to quantify vertices' centrality.

Meanwhile, in order to test our approach's accuracy in revealing topological structure, we calculate the betweenness centrality of each vertex, says $B_{c}(v)$ for vertex $v$, in an undirected network as the following formula [4],

$B_{c}(v)=\frac{2}{(N-1)(N-2)} \sum_{s \neq v \neq d} \frac{n_{s d}(v)}{n_{s d}}$

where $n_{s d}$ is the total number of shortest paths from vertex $s$ to vertex $d, n_{s d}(v)$ is the number of those paths pass through $v$. We calculated the betweenness centrality $B_{c}$ and the probabilities $P_{10}$ and $P_{20}$ for each vertex respectively. For some known vital vertices (Table 1), for example, vertex 23 in the DSN and vertex 64 in the PPIN, we found that vertices with large betweenness centrality have large $P_{10}$ and $P_{20}$, i.e., those vital vertices have large probability of being identified by means of our approach. It is surprising that there also exist some exception vertices (such as vertex 21 and vertex 24 in the DSN) have large dynamical centrality but have small betweenness centrality due to the difference between our approach and the betweenness centrality index. In the betweenness centrality index, the betweenness centrality is calculated based on the global information about the topological structure which is hidden in a black box. While in our dynamical approach, the dynamical centrality is calculated just only based on vertices' status and their local dynamical abilities. Although there exists a clear difference between the definitions of our approach and the betweenness centrality index, our approach has a satisfactory performance to identify vital vertical with large betweenness centrality, which shows the novelty of our approach to reveal vertices' topological property in complex networks.

\section{Application}

Due to our approach's satisfactory performance in identifying overlapping vertices, we shed light on the application of our approach to detect community in complex networks, where vertices are divided into groups. Vertices are more tightly connected with each other in the same group, meanwhile there also exist some overlapping vertices belonging to more than one group. Further, in order to quantify the validity of possible subdivisions, we use the concept of modularity $Q$ as a function form $[32,33]$

$Q=\sum_{i}\left(e_{i i}-a_{i}^{2}\right)$

where $a_{i}=\sum_{j} e_{i j}$ is the total fraction of links with one vertex in community $i$ in network and $e_{i j}$ is the fraction of all links that link vertices in community $i$ to vertices in community $j$. The modularity $Q$ is a practical index to assess a given division into any number of communities for a given network. Larger value of $Q$ indicates stronger community structure [34].

Combining with the process of quantifying vertices' centrality and the process of community detection, we introduce two different times: the dynamical time $t$ and the detecting action $n$. The algorithm of identifying community using the dynamical approach to quantify vertices' centrality is simply stated as follows. As mentioned above, each vertex denoted by $i$ is encoded a phase oscillator with its natural frequency $\omega_{i}$ and phase $\theta_{i}$. Step 1 . The 


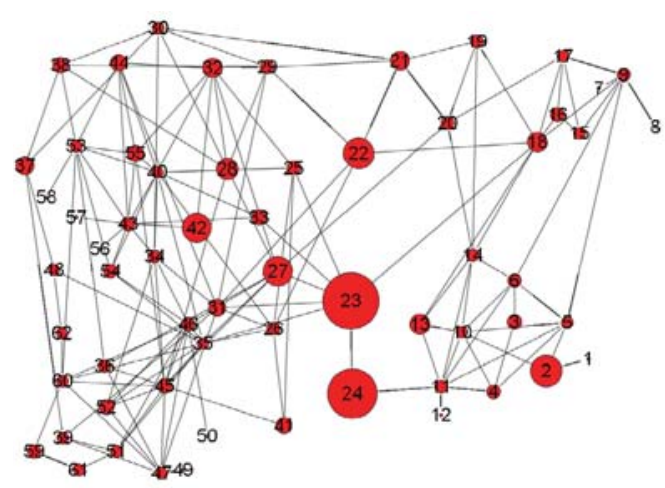

(a)

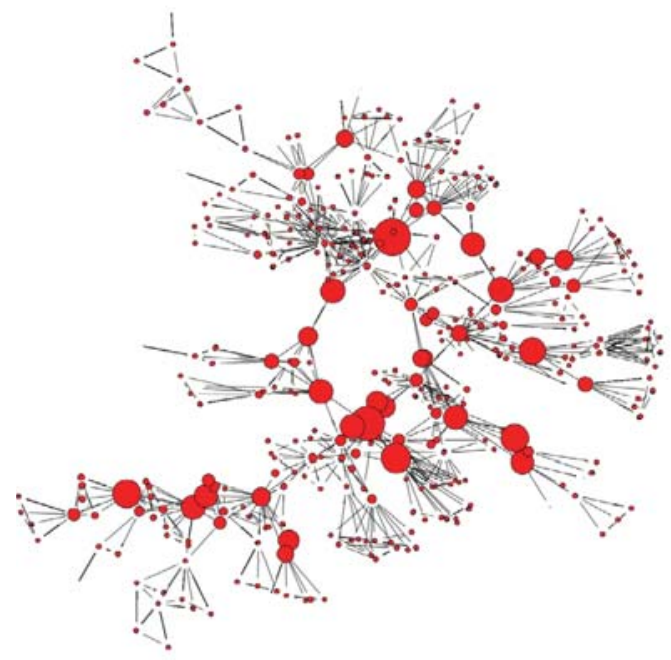

(c)

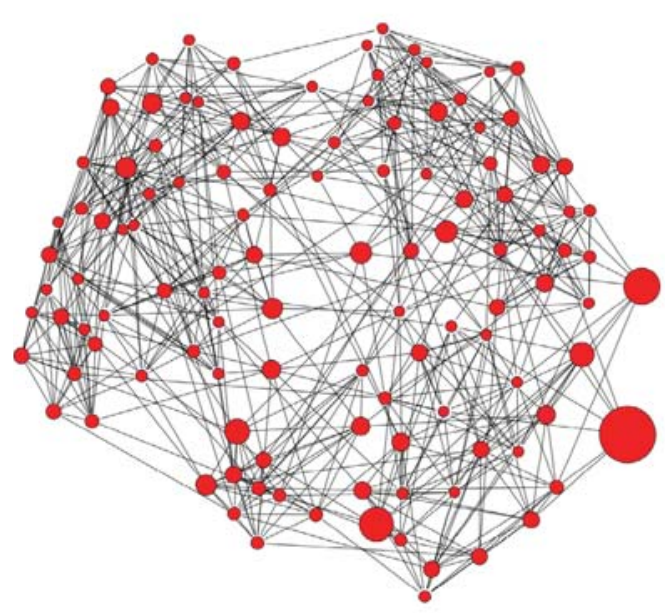

(e)

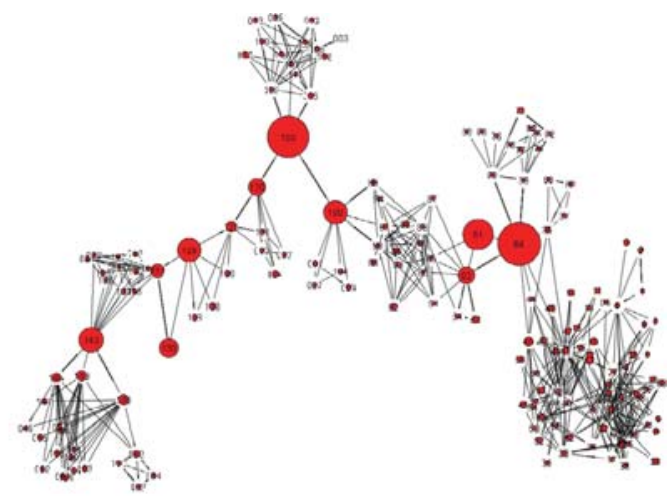

(b)

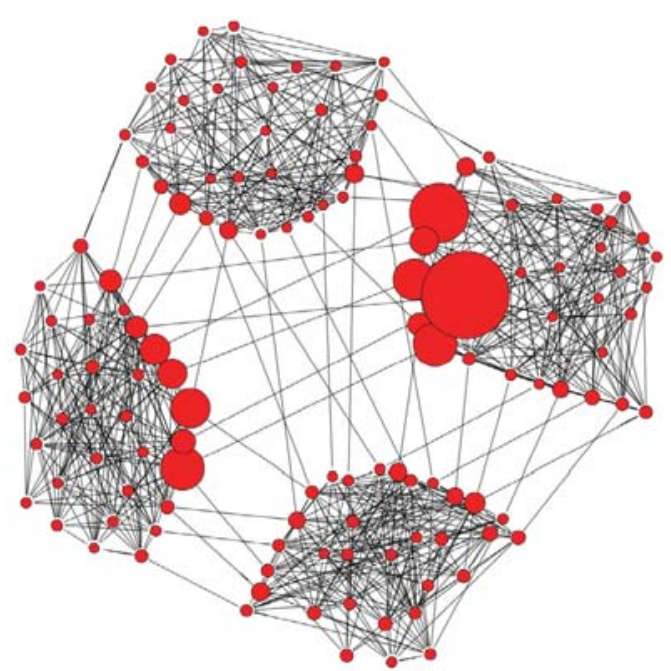

(d)

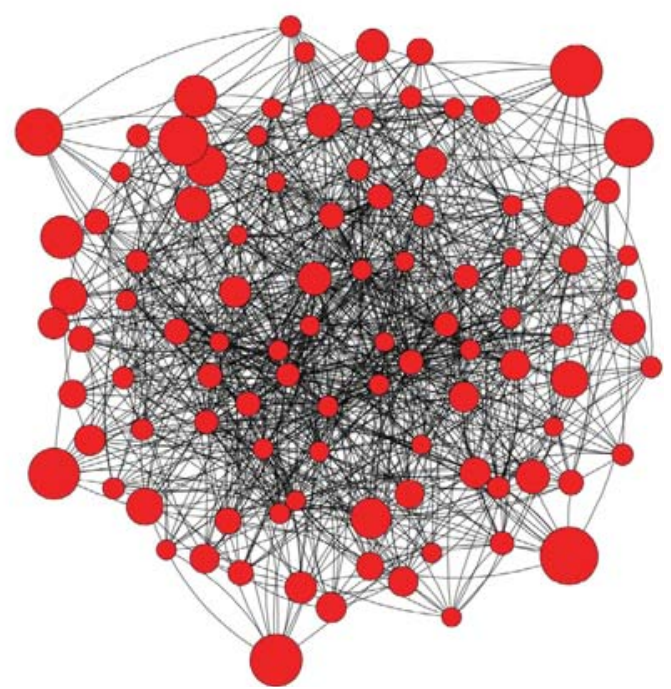

(f)

Fig. 2. (Color online.) An intuitive display of $\left\langle\eta_{i}^{\prime}\right\rangle_{t}$ of each oscillator in (a) the DSN, (b) the PPIN, (c) the network of coauthorships between scientists whose research centers on the top of networks, (d) the benchmark network, (e) the college football network and (f) the BA scale-free network at the dynamical time $t=300$. The number labeled on each node is the numerical code of the corresponding node in (a) and (b) and the value of each oscillator's $\left\langle\eta_{i}^{\prime}\right\rangle_{t}$ (for all $i$ ) is proportional to the radius of the corresponding circle.

dynamical process of the Kuramoto model. Each oscillator, says $i$, updates its phase $\theta_{i}(t)$ according to Eq. (1), and then we calculate $\left\langle\eta^{\prime}\right\rangle_{t} \mathrm{~s}$ for all oscillators at the dynamical time $t$. Step 2. The detecting process of community. All the oscillators are sorted as a decreasing order according to their $\left\langle\eta^{\prime}\right\rangle_{t}$ s. And then, all the os- cillators are operated in turn to identify community: for the $n$-th oscillator, we keep the only one link that has the largest number of common neighbors and delete all the other links, and calculate the modularity $Q_{t}(n)$ at the detecting action $n$, till all the oscillators are operated. Step 3. All the deleted links are repaired and 

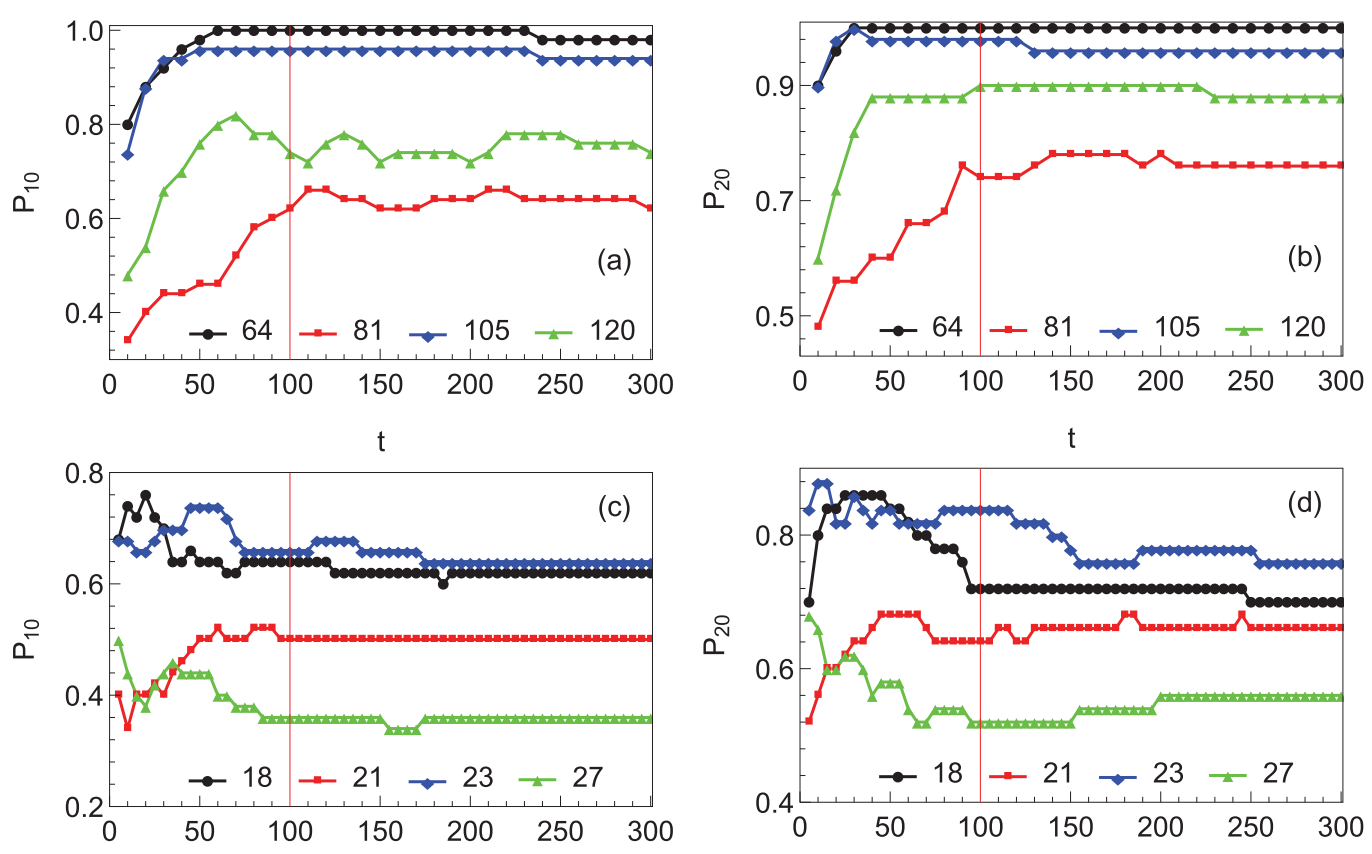

$\mathrm{t}$

Fig. 3. (Color online.) The probability $\left(P_{10}\right)$ that each given oscillator falls in the top ten evolves as dynamical time $t$ elapses in (a) the PPIN and (c) the DSN. And the probability $\left(P_{20}\right)$ that each given oscillator falls in the top twenty evolves as dynamical time $t$ elapses in (b) the PPIN and (d) the DSN. The codes of these oscillators are the same as that in Fig. 2(a) and Fig. 2(b), respectively. Red lines are guides to the eye.

(2)

Table 1

This table shows the probabilities that some known vital oscillators (codes: No.) fall in the top ten $\left(P_{10}\right)$ and in the top twenty $\left(P_{20}\right)$ in the PPIN and in the DSN - at the dynamical time $t=300 . B_{c}$ is the score of betweenness centrality for the corresponding vertex. The codes of these oscillators (No.) are the same as that in Fig. 2(a) and Fig. 2(b), respectively.

\begin{tabular}{lllllllll}
\hline & PPIN & & & & \multicolumn{3}{c}{ DSN } \\
\cline { 1 - 2 } \cline { 6 - 7 } No. & $P_{10}$ & $P_{20}$ & $B_{C}$ & & No. & $P_{10}$ & $P_{20}$ & $B_{c}$ \\
\hline 64 & $98 \%$ & $100 \%$ & 0.565 & & 24 & $66 \%$ & $74 \%$ & 0.076 \\
105 & $94 \%$ & $96 \%$ & 0.510 & & 23 & $64 \%$ & $76 \%$ & 0.250 \\
100 & $78 \%$ & $94 \%$ & 0.497 & & 18 & $62 \%$ & $70 \%$ & 0.209 \\
120 & $74 \%$ & $88 \%$ & 0.394 & & 20 & $54 \%$ & $62 \%$ & 0.122 \\
129 & $70 \%$ & $80 \%$ & 0.333 & & 21 & $50 \%$ & $66 \%$ & 0.034 \\
143 & $54 \%$ & $76 \%$ & 0.229 & & 27 & $36 \%$ & $56 \%$ & 0.146 \\
\hline
\end{tabular}

the both processes are repeated from step 1 as the dynamical time $t$ elapses. Note that each link is operated one time whether it is deleted or not and all the mutual interactions between oscillators are not destroyed during the detecting process of community.

We analyze the evolution of the modularity $Q_{t}(n)$ as a function of the detecting action $n$ at each dynamical time $t$ in the PPIN. In Fig. 4, we plot the relationship between $Q_{t}(n)$ and the detecting action $n$ at the dynamical time $t=10,20,50$ and 100, respectively. We find that $Q_{t}(n)$ reaches its maximum value at about $n=10$ in the PPIN. Namely, there exists a peak with the maximum value of $Q_{t}(n)$ when we operate the community detecting process on the top ten oscillators, which shows that our dynamical approach has its better performance in identifying the overlapping oscillators. Another surprising result is that the modularity $Q$ reaches its maximum value at $t=20$ during the dynamical process of quantifying vertices' centrality, which shows that our dynamical approach has high efficiency although there exists some internal initial noise. Note that our results reveal the positive role of dynamics process in reconstructing the topological structure of those interaction units in complex networks. Since structural information is often hidden in the dynamical behavior of the vertices in an unknown manner $[16,35,36]$, we recognize that vertices with

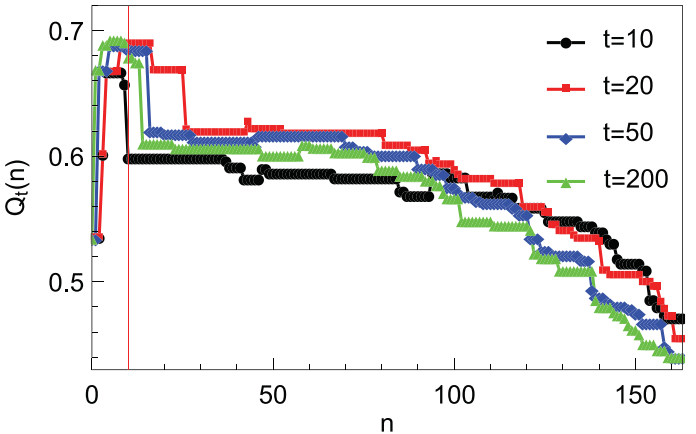

Fig. 4. (Color online.) The modularity $Q$ evolves as a function of the detecting action $n$ in the protein-protein interaction network when oscillators evolve at the dynamical time $t=10,20,50$ and 100 , respectively. The red line shows that the value of the modularity $Q$ when we operate the top ten oscillators using the detecting process.

the similar dynamical properties have a large probability to have a similar topological structure.

\section{Conclusion}

In summary, we proposed a dynamical approach to identify vertices' centrality during the synchronization process of the Kuramoto model. Different from some previous literatures, the novelty of our dynamical approach is that vertex's centrality is quantified based on the DSA. We analyzed the robustness and efficiency of our dynamical approach through applying it to detect community in the PPIN and the DSN. Furthermore, we found that our approach has a better performance to identify the weak links, which end with vertices with a higher dynamical centrality. Namely, the weak links connect the overlapping vertices in complex networks with a clearly community structure. Our present work provides a new perspective and tools to understand the bridging role of heterogeneity (i.e., difference) in revealing the connection between the dynamical behavior and the underlying structure of elementary units in complex networks. 


\section{Acknowledgements}

L. Guo and W.Y. Zhang wish to acknowledge the financial support by the China Scholarship Council under Grant Nos. 201606415063 and 201406340126.

\section{References}

[1] G. Bianconi, Interdisciplinary and physics challenges of network theory, Europhys. Lett. 111 (2015) 56001.

[2] R. Albert, A.-L. Barabási, Statistical mechanics of complex networks, Rev. Mod. Phys. 74 (2002) 47.

[3] S. Boccaletti, V. Latora, Y. Moreno, M. Chavez, D.-U. Hwang, Complex networks: structure and dynamics, Phys. Rep. 424 (2006) 175-308

[4] L.Y. Lü, D.B. Chen, X.L. Ren, Q.M. Zhang, Y.C. Zhang, T. Zhou, Vital nodes identification in complex networks, Phys. Rep. 650 (2016) 1-63.

[5] I. Psorakis, S. Roberts, M. Ebden, Overlapping community detection using Bayesian non-negative matrix factorization, Phys. Rev. E 83 (2011) 066114.

[6] Z.-Y. Zhang, Y. Wang, Y.-Y. Ahn, Overlapping community detection in complex networks using symmetric binary matrix factorization, Phys. Rev. E 87 (2013) 062803.

[7] S. Fortunato, D. Hric, Community detection in networks: a user guide, Phys. Rep. 659 (2016) 1-44.

[8] Y. Chen, X. Wang, X. Xiang, B. Tang, Q. Chen, S. Fan, J. Bu, Overlapping community detection in weighted networks via a Bayesian approach, Physica A 468 (2017) 790-801.

[9] J.G. Liu, J.H. Lin, Q. Guo, T. Zhou, Locating influential nodes via dynamicssensitive centrality, Sci. Rep. 6 (2016) 21380.

[10] Z.M. Ren, A. Zeng, D.B. Chen, H. Liao, J.G. Liu, Iterative resource allocation for ranking spreaders in complex networks, Europhys. Lett. 106 (2014) 48005.

[11] H. Liao, M.S. Mariani, M. Medo, Y.-C. Zhang, M.-Y. Zhou, Ranking in evolving complex networks, Phys. Rep. 689 (2017) 1-54.

[12] G. Jeon, J. Park, Impact of biased scores on ranking in bipartite competition networks and inference of modular structure via generalized modularity, Europhys. Lett. 117 (2017) 48005.

[13] H. Liao, A Zeng M.Y Zhou, R. Mao, B.-H, Wang Information mining in weighted complex networks with nonlinear rating projection, Commun. Nonlinear Sci. Numer. Simul. 51 (2017) 115-123.

[14] Z.Y. Wang, J.T. Han, J. Zhao, Identifying node spreading influence for tunable clustering coefficient networks, Physica A 486 (2017) 242-250.

[15] K. Klemm, M.Á. Serrano, V.M. Eguíluz, M.S. Miguel, A measure of individual role in collective dynamics, Sci. Rep. 2 (2012) 292.

[16] J.W. Li, Z.S. Shen, W.X. Wang, C. Grebogi, Y.C. Lai, Universal data-based method for reconstructing complex networks with binary-state dynamics, Phys. Rev. E 95 (2017) 032303.
[17] F. Alderisio, G. Fiore, M. di Bernardo, Reconstructing the structure of directed and weighted networks of nonlinear oscillators, Phys. Rev. E 95 (2017) 042302

[18] J.G. Liu, X.L. Liu, Q. Guo, J.T. Han, Identifying the perceptive users for online social systems, PLoS ONE 12 (2017) e0178118.

[19] J. G.-Gardeñes, Y. Moreno, A. Arenas, Paths to synchronization on complex networks, Phys. Rev. Lett. 98 (2007) 034101

[20] S. Fortunato, Community detection in graphs, Phys. Rep. 486 (2010) 75-174

[21] J.J. Crofts, M. Forrester, R.D. O'Dea, Structure-function clustering in multiplex brain networks, Europhys. Lett. 116 (2016) 18003.

[22] A. Arenas, A. Díaz-Guilera, C.J. Pérez-Vicente, Synchronization reveals topological scales in complex networks, Phys. Rev. Lett. 96 (2006) 114102.

[23] M. De Domenico, Diffusion geometry unravels the emergence of functional clusters in collective phenomena, Phys. Rev. Lett. 118 (2017) 168301.

[24] D. Li, I. Leyva, J.A. Almendral, I. S.-Nadal, J.M. Buldú, S. Havlin, S. Boccaletti, Synchronization interfaces and overlapping communities in complex networks, Phys. Rev. Lett. 101 (2008) 168701.

[25] F.A. Rodrigues, T.K.DM. Peron, P. Ji, J. Kurths, The Kuramoto model in complex networks, Phys. Rep. 610 (2016) 1-98

[26] A. Arenas, A. Díaz-Guilera, J. Kurths, Y. Moreno, C.S. Zhou, Synchronization in complex networks, Phys. Rep. 469 (2008) 93-153.

[27] D. Lusseau, K. Schneider, O.J. Boisseau, P. Haase, E. Slooten, S.M. Dawson, The bottlenose dolphin community of Doubtful Sound features a large proportion of long-lasting associations, Behav. Ecol. Sociobiol. 54 (2003) 396-405.

[28] M.E.J. Newman, Finding community structure in networks using the eigenvectors of matrices, Phys. Rev. E 74 (2006) 036104.

[29] M. Girvan, M.E.J. Newman, Community structure in social and biological networks, Proc. Natl. Acad. Sci. USA 99 (2002) 7821-7826.

[30] A.-L. Barabási, R. Albert, Emergence of scaling in random networks, Science 286 (1999) 509-512.

[31] P.F. Jonsson, T. Cavanna, D. Zicha, P.A. Bates, Cluster analysis of networks generated through homology: automatic identification of important protein communities involved in cancer metastasis, BMC Bioinform. 7 (2006) 2.

[32] A. Clauset, M.E.J. Newman, C. Moore, Finding community structure in very large networks, Phys. Rev. E 70 (2004) 066111

[33] L. Guo, X. Cai, Emergence of community structure in the adaptive social networks, Commun. Comput. Phys. 8 (2010) 835-844.

[34] X. Zhang, M.E.J. Newman, Multiway spectral community detection in networks, Phys. Rev. E 92 (2015) 052808.

[35] Y. Wang, J. Wang, H. Liao, H. Chen, An efficient semi-supervised representatives feature selection algorithm based on information theory, Pattern Recognit. 61 (2017) 511-523.

[36] Y. Chen, Z.Y. Zhang, T.Y. Chen, S.H. Wang, G. Hu, Reconstruction of noise-driven nonlinear networks from node outputs by using high-order correlations, Sci. Rep. 7 (2017) 44639. 